\title{
Base de datos de eventos solares observados desde la Plaza del Sol del Parque Arqueológico de Copán Ruinas
}

\author{
María Cristina Pineda de Carías* \\ Santos Vito Véliz*
}

\section{RESUMEN}

El objetivo principal de este trabajo ha sido diseñar y conformar una base de datos a partir del uso del material fotográfico recolectado entre 1998 y 2008 por nosotros, como Grupo de Arqueoastronomía del Observatorio Astronómico Centroamericano de Suyapa de la Universidad Nacional Autónoma de Honduras. En este trabajo describimos las características de la base de datos relacional que contiene 2,467 fotografías y 8 tablas de atributos con fechas y lugares desde donde se realizaron las observaciones de las salidas y puestas del Sol, o para diferentes horas del día para conocer el tamaño y la dirección de las sombras de la Estela D, reloj solar del Parque Arqueológico de Copán Ruinas. La utilización del software de dominio público Picasa3 en combinación con hojas de cálculo de Excel facilita que cualquier usuario, en especial de la comunidad de investigadores y personas estudiosas de la Arqueoastronomía, aproveche esta información para comprender cómo se realizaban las observaciones astronómicas en Copán Ruinas o para generar nuevas líneas de investigación en Arqueoastronomía.

Palabras clave: Arqueoastronomía, observaciones solares, Parque Arqueológico Copán Ruinas, base de datos.

\section{ABSTRACT}

The main objective of this work has been to design and form a database using photographic material collected between 1998 and 2008 by us as a group of Archaeoastronomy of the Central American Suyapa Astronomical Observatory of the National Autonomous University of Honduras. In this paper we describe the characteristics of the relational database containing 2.467 photographs and 8

\footnotetext{
* María Cristina Pineda de Carías, mcarias@cablecolor.hn

* Santos Vito Véliz, vitoveliz@yahoo.com

Facultad de Ciencias Espaciales, Observatorio Astronómico Centroamericano de Suyapa,

Universidad Nacional Autónoma de Honduras.
} 
attribute tables with dates and places where observations were made at sunrises and sunsets, or for different times of day from the size and direction of the shadows of Stela D, sundial of the Archaeological Copan Ruins Park. The use of Picasa3 open source software in combination with Excel spreadsheets easier for anyone, especially the community of researchers and scholars of Archaeoastronomy, use this information to understand how astronomical observations were made in Copan or to generate new lines of research in Archaeoastronomy.

Keywords: Archaeoastronomy, solar observations, Archaeological Copan Ruins Park, database. 


\section{INTRODUCCIÓN}

Desde el año 1998 hasta el presente, los autores de este trabajo conformamos el Grupo de Arqueoastronomía del Observatorio Astronómico Centroamericano de Suyapa de la Universidad Nacional Autónoma de Honduras (OACS/UNAH). Desde esa fecha hemos venido realizando observaciones astronómicas e investigaciones arqueoastronómicas en el Parque Arqueológico de Copán Ruinas, las cuales han puesto en evidencia los conocimientos que los mayas tenían acerca de los movimientos del Sol y cómo estos pudieron ser utilizados para la construcción y orientación de estructuras, graderías y monumentos, así como para medir el tiempo. Estos trabajos se han concentrado específicamente en el sector norte de la Plaza Principal del Parque Arqueológico de Copán Ruinas, motivo por el cual propusimos llamar a este sector Plaza del Sol.

A lo largo de casi una década de trabajo, hemos logrado significativos avances en nuestras investigaciones, algunas de las cuales aparecen publicadas en la Revista Yaxkin del Instituto Hondureño de Antropología e Historia (Pineda de Carias, Véliz y Agurcia Fasquelle 2002); otras las hemos presentado en numerosos seminarios, congresos y conferencias realizadas en la UNAH y en diferentes instituciones nacionales e internacionales. Por la forma en que fue variando nuestro objeto de estudio, los resultados podemos agruparlos en cuatro grandes categorías:

\section{De los alineamientos (1998-2000)}

Cuando descubrimos numerosos alineamientos de estelas y orientación de estructuras y graderías, asociados con las salidas o las puestas del Sol en las fechas de los equinoccios, los solsticios y los pasos del Sol por el cenit de Copán Ruinas.

\section{Sobre el Grande y Complejo Plan de 18 Conejo para la Plaza del Sol (2000- 2002)}

Cuando presentamos, a partir de los resultados de la etapa anterior y su ubicación en el tiempo, el descubrimiento de que la colocación de las estelas y la construcción de las graderías y estructuras que le dan forma a la Plaza Principal, estaban amarradas con alineamientos asociados con las salidas y puestas del Sol, ocurridas en equinoccios, solsticios y pasos del Sol por el cenit de Copán Ruinas. 


\section{Estelas como instrumentos de observación (2002-2006)}

Una etapa en la que logramos demostrar cómo las estelas fueron utilizadas para señalar la posición del Sol sobre el horizonte, en fechas de eventos especiales, ocurridas dentro del ciclo haab del calendario maya. Durante este período hicimos gestiones para conseguir el emplazamiento de una réplica de la Estela $B$ en los predios del OACS/UNAH en la Ciudad Universitaria de Tegucigalpa, para ser utilizada con las mismas funciones que la original Estela B de Copán Ruinas.

\section{Estela D: Reloj Solar de Copan Ruinas (2007)}

Cuando descubrimos que esta Estela $D$ y su altar, junto con las graderías del sector norte de la Plaza del Sol, eran un reloj solar que utilizaban los mayas de Copán Ruinas para medir las divisiones del día y del año trópico.

Producto de estas investigaciones fue la generación de varios miles de fotografías de las salidas y puestas del Sol y de las sombras de la Estela $D$, tomadas desde la Plaza del Sol, durante las observaciones astronómicas realizadas en la fecha de los equinoccios, solsticios y pasos del Sol por el cenit de Copán Ruinas. La acumulación de esta enorme cantidad de datos generó también la necesidad de almacenarlos y guardarlos para preservarlos con miras hacia su posterior uso. Fue así como identificamos nuestro problema de estudio, preguntándonos: ¿Cómo mejorar y aprovechar al máximo la información fotográfica generada como Grupo de Arqueoastronomía del OACS/UNAH sobre la Plaza del Sol de Copán? ¿Cómo organizar los datos de manera que pudieran ser aprovechados por otras personas interesadas en realizar investigaciones arqueoastronómicas? ¿Cómo poner a la disposición de docentes y estudiantes todo este material documental para su estudio y comprensión de la cultura maya?

Para resolver este problema identificamos como el objetivo de este trabajo el diseño de un sistema de base de datos relacional que integrara 2,467 fotografías con software de computación para almacenamiento, recuperación, despliegue y consulta, de manera aislada o conjunta de las fotografías.

Con esta base de datos, la comunidad científica y los estudiosos de los temas de Arqueoastronomía tendrán una herramienta poderosa que les permita, de manera eficiente, acceder a los datos contenidos en las fotografías. El uso de una página web hará posible que, vía Internet, investigadores nacionales e internacionales se beneficien de los resultados obtenidos por el Proyecto de Arqueoastronomía Maya delOACS/UNAH. 


\section{METODOLOGÍA}

El presente trabajo lo desarrollamos en seis etapas:

1. Localización y recolección de las fotografías obtenidas en el Proyecto de Arqueoastronomía Maya entre los años 1998 y 2007.

2. Evaluación del estado de las fotografías y selección de las que utilizaríamos en este Proyecto.

3. Diseño de un Sistema de Base de Datos (SBD) para integrar las fotografías (los datos) y los programas de computación para su manejo y visualización.

4. Almacenaje de las fotografías e introducción de los datos de cada fotografía a la base de datos.

5. Instalación del software para visualización de las fotografías.

6. Diseño de página web para poner a disposición de usuarios potenciales el SBD.

\section{RESULTADOS}

\section{Selección de las fotografías para uso del Proyecto}

Al inicio de este trabajo, localizamos y recolectamos cerca de diez mil fotografías tomadas entre 1998 y 2007.

Del estudio de las fechas en que fueron tomadas estas fotografías logramos identificar que, las que se tomaron en los años más tempranos se encontraban impresas y pegadas en quince álbumes, por lo que una primera tarea fue digitalizarlas. Las del tercio intermedio habían sido tomadas con una cámara analógica pero que fueron digitalizadas al momento de ser reveladas, encontrándose disponibles unas en diferentes CDs, y otras en diferentes computadoras del OACS. Las del tercio más reciente todas fueron tomadas con cámaras digitales por lo que las mismas estaban almacenadas en diferentes computadoras del OACS.

Para evaluar el contenido de la información disponible en cada fotografía, tomamos como criterio principal la evidencia para soportar y documentar observaciones astronómicas que fueron hechas a las salidas o a las puestas del Sol en las fechas de eventos tales como equinoccios, solsticios y pasos del Sol por el cenit de Copán Ruinas o porque recogían la sombra proyectada por la Estela $D$ en diferentes horas del día. Fue después de una selección rigurosa que dejamos reducido el número de las fotografías para uso del proyecto en 2,467. Para los años 1998, 1999 y 2004 no se seleccionó ninguna fotografía. 


\section{Diseño de la Base de Datos}

Como método eficiente para manipular el enorme volumen de fotografías, se buscó diseñar un sistema de base de datos consistente de toda la colección de las fotografías más un conjunto de programas de computación que permitiera a cualquier usuario acceder a los datos. Este sistema se instalaría y manejaría en una computadora personal, dedicada exclusivamente para este trabajo.

El modelo de la base de datos seleccionado fue relacional, por lo que se tuvo que diseñar tablas para presentar tanto los datos de cada fotografía como las relaciones entre ellas. Cada tabla debía tener una fila para registro de cada fotografía y varias columnas para los datos de cada fotografía, teniendo cada una de estas columnas un nombre único para representar también un atributo específico de cada fotografía. Sería entonces un modelo basado en registros de formato fijo (Silberschatz, Karth y Saudarshan 2006).

Los datos a recoger como atributos de cada fotografía serían los siguientes:

1. AÑO: El año en que fue tomada la fotografía.

2. EVENTO: El tipo de evento solar alrededor del cual fue tomada la fotografía. Estos eventos podían ser clasificados en:

- EQPV: Equinoccio de Primavera

- 1PSC: Primer paso del Sol por el cenit

- SOLV: Solsticio de Verano

- 2PSC: Segundo paso del Sol por el cenit.

- EQOT: Equinoccio de Otoño

- SOLI: Solsticio de Invierno.

3. FECHA: Para documentar el día y mes de la observación por evento.

4. Para cada día de trabajo alrededor de un evento solar, también había que documentar si las fotografías habían sido tomadas para observar:

- SALIDAS: Salidas del Sol.

- PUESTAS: Puestas del Sol.

En cada caso se indica la dirección desde donde y hacia donde se hizo la observación.

- ESTELAD: Dirección y tamaño de la sombra.

Distinguiendo entre la mañana, el medio día y la tarde.

5. En un mismo día de trabajo, y para las salidas o puestas del Sol, o para las sombras de la Estela $D$, las fotografías debían almacenarse en secuencia, es decir, por el orden en que fueron tomadas. 


\section{Digitalización y almacenamiento de las fotografías}

Como una consecuencia de las características del diseño de la base de datos, en una necesidad se convirtió la digitalización primero y luego la asignación de un nombre único para cada una de las 2,467 fotografías seleccionadas. El nombre de cada fotografía directamente sugería: el año, el tipo de evento, la fecha en que fue tomada, el número de la secuencia de toma de la salida, la puesta o la sombra de la Estela D. Por ejemplo: 2000_2PSC_12Ago_140E, era el nombre de una fotografía tomada el 12 de Agosto de 2000 , alrededor del día del segundo paso del Sol por el cenit para documentar la sombra de la Estela $\mathrm{D}$, teniendo como número de secuencia asignado el 140.

Las 2,467 fotografías se almacenaron en 62 carpetas que también tenían un nombre único, sugerente de la fecha y el evento en que fueron tomadas. Por ejemplo, 2000_2PSC_12Ago_EstelaD, era el nombre de la carpeta con el grupo de fotografías tomadas el 12 de Ägosto de 2000, alrededor del segundo paso del Sol por el cenit para documentar el tamaño de las sombras de la Estela D. Para el período estudiado del 2000 al 2008, se almacenaron: 176 fotografías del año 2000, organizadas en 11 archivos; 339 fotografías del 2001, organizadas en 11 archivos; 467 fotografías del año 2002, organizadas en 16 archivos; 145 fotografías del año 2003, organizadas en 5 archivos; 126 fotografías del 2005, organizados en 2 archivos; 63 fotografías del 2006, organizadas en 3 archivos; 912 fotografías del 2007, organizadas en 13 archivos; y 239 fotografías del 2008, organizadas en un único archivo.

\section{Tabulación de los datos de las fotografías}

Ocho tablas de Excel fueron elaboradas para documentar, por cada uno de los años del 2000 al 2008 (exceptuando el 2004), los atributos de cada fotografía. Cada tabla se conformó con tantos registros como fotografías se habían seleccionado por año. Los atributos se organizaron en 10 columnas tituladas:

1. CARPETA: para incluir el nombre único de la carpeta donde se archivó la fotografía, por ejemplo: 2006 SOLI_21Dic_EstelaD.

2. No. ORDEN: que incluye de manera secuencial la numeración de las fotografías de la Carpeta.

3. No. FOTO: que contiene el nombre único de la fotografía con un hipervínculo haciala propia foto, como por ejemplo: 2006_SOLI_21Dic_017E.

4. AÒO: que se refiere al año en que se tomó la fotografía.

5. EVENTO: que se refiere al evento solar asociado con la campaña de observación 
en la que se tomó la fotografía.

6. FECHA: se refiere al día, mes y año en que se tomó la fotografía.

7. HORA: se refiere a la hora local (horas y minutos) en que se tomó la fotografía; se aclara que este campo no se pudo completar porque este dato no estaba disponible para todas las fotografías.

8. DESCRIPCION: este campo describe, para las fotografías de las salidas o de las puestas del Sol, el lugar desde donde y hacia donde se dirigía la línea de observación; en el caso de las fotografías de las sombras de la Estela D, se indica la cara de la Estela que se está mirando y el intervalo del día correspondiente, según se cree marca la propia Estela $\mathrm{D}$ en su función de reloj solar (en tiempo solar: mañana: desde la salida del Sol hasta alrededor de las 8:00 horas, antes de medio día: entre las 8:00 y unos minutos antes de las 12:00 horas, medio día: alrededor de las 12:00 horas cuando la Estela D y su sombra están alineadas, después de medio día: desde unos minutos después de las 12:00 a las 16:00 horas, tarde: entre las 16:00 horas y la puesta del Sol.

9. REFERENCIA: Nombre de la Carpeta que sugiere la fuente donde originalmente se encontraron las diferentes fotografías, por ejemplo: Álbum 10 que contiene algunas de las fotografías impresas de los años 2000, 2001 y 2002.

10. No. CORRIDA: que se refiere a la numeración corrida de todas las fotografías tomadas en un año determinado. A manera de ejemplo, en la Figura 1 se muestra la Hoja de Cálculo de Excel que contiene los atributos de las fotografías tomadas el año 2003.

Hipervínculos se establecieron para enlazar el nombre de cada fotografía con la dirección de archivo de la fotografía donde se encontraba en el disco duro de la computadora. En la Figura 1 se muestra el ejemplo de una de las ocho tablas preparadas.

\section{Sistema de visualización de las diferentes fotografías}

El sistema de base de datos fue diseñado para ver cada fotografía como una vista; fue por ello que el software de dominio público Picasa3 de Google Earth (http://picasa.google.com) se escogió para organizar, clasificar, editar, buscar, recuperar y manipular las fotografías puestas a disposición de los usuarios. De esta manera, los datos con que fueron agregados a la computadora las diferentes fotografías, automáticamente permitían que el software los organizara para ser visualizados. Así, a voluntad del usuario podían desplegarse, por año, tipo de evento astronómico, fecha en que fueron tomadas las fotografías y grupo de observación durante el día. Los datos, en este caso las fotografías, podían ser desplegadas por contenido de las carpetas, o como un show de diapositivas o como 
un arreglo por tipo de dato sobre una línea de tiempo.

Al instalar el software Picasa3, automáticamente se escanearon todos los archivos grabados en el disco duro de la computadora. Al abrir Picasa3, aparecieron las carpetas organizadas en secuencia temporal, mostrando para cada año todas las carpetas de los eventos solares en que se ha agrupado las fotografías. A manera de ejemplo, en la Figura 2 se muestra la pantalla de la Biblioteca de Archivos de Picasa3. En el lado izquierdo se pueden ver las carpetas correspondientes a los años 2002, 2003, 2005, 2006 y 2007. Al lado derecho, en pequeños iconos, aparecen las fotografías contenidas en la Carpeta 2007 SOLI_22Dic_EstelaD, secuencia en la que cada fotografía aparece rotulada con su nombre. En la parte inferior, se muestra el número de fotografías que contiene la Carpeta, la fecha en que fueron tomadas y el tamaño que los archivos ocupan en el disco.

\section{Presentación de datos por Internet y resguardo}

Para la gestión del sistema de base de datos (SBD) se diseñó una página de Internet accesible desde la página web del Observatorio Astronómico Centroamericano de Suyapa (http://ww.oacs-unah.edu.hn). En esta página se presentan los datos de manera general y se indica a los usuarios potenciales la forma de contactar a los Profesores Coordinadores del Proyecto de Arqueoastronomía del OACS/UNAH.

En una computadora dedicada se instaló el SBD, es decir, la base de datos que contiene todas las fotografías, el software Picasa3 y el software Excel de Microsoft. Archivos de respaldo se prepararon para evitar alguna eventual pérdida. De igual manera se preparó un documento impreso, describiendo la Base de Datos. Las fotografías seleccionadas para formar parte de la base de datos son aquellas que tienen un valor científico en cuanto al dato capturado, por lo que todas en su conjunto están en trámite de registro en la Dirección de Propiedad Intelectual del Instituto de la Propiedad de Honduras.

\section{DISCUSIÓN Y CONCLUSIONES}

La base de datos que aquí se presenta por primera vez pone a la disposición de la comunidad científica, nacional e internacional, abundante material fotográfico que hace posible entender y apreciar cómo se piensa que los mayas de Copán pudieron haber observado la ocurrencia de equinoccios, solsticios y pasos del Sol por el cenit de Copán Ruinas, desde la Plaza del Sol. 
Se reconoce que la colección no puede considerarse suficientemente completa y habrá algunas ventanas que precisamente serán las que darán lugar a que nuevos trabajos de investigación se puedan realizar. Se estimulará, por tanto, a los estudiosos de laArqueoastronomía, un campo que formalmente ya se ha iniciado en la UNAH, para que utilicen y saquen el mayor provecho del material recopilado. Un trabajo de esta naturaleza nunca antes se había presentado por lo que, agregado al valor de los propios datos, está la posibilidad de mostrar al sitio de Copán Ruinas como un lugar especial adonde seguramente los gobernantes de las diferentes dinastías se reunían con sus gobernados para realizar observaciones astronómicas y ceremonias especiales en los días de eventos solares de interés. La abundancia de los datos presentados, por ella misma, sirve de argumento central para corroborar por qué los autores hemos propuesto bautizar al sector norte de la Plaza Principal del Parque Arqueológico como Plaza del Sol.

No hay duda que la base de datos con la que ahora se dispone, manejada con las nuevas tecnologías, no sólo nos permite visualizar mejor los diferentes escenarios del período que vivieron los mayas, sino que también nos ayuda a comprender cabalmente cómo en realidad se desempeñaban en su diario quehacer. La oportunidad de revisar, estudiar y analizar las fotografías de esta base de datos seguramente permitirá que algunos investigadores incursionen en la línea de querer entender qué tipo de ritos o ceremonias realizaban los mayas en las fechas de eventos solares. Los niveles de organización y opciones de manipulación de los datos para hacer comparaciones de fotografías de diferentes fechas, de diferentes eventos u otras que puedan parecer de interés a un usuario y todo lo que el SBD elaborado permite, sin lugar a dudas es el mayor resultado alcanzado con este trabajo.

\section{AGRADECIMIENTOS}

Se agradece a la Dirección de Investigación Científica de la Universidad Nacional Autónoma de Honduras por haber otorgado la Beca Básica No. 05-BBI-2007 que hizo posible la realización de este trabajo. Se agradece también al personal de Arqueoastronomía del OACS/UNAH, especialmente a Bertilio Amaya por haber colaborado en la digitalización de algunas de las fotografías.

\section{BIBLIOGRAFÍA}

Pineda de Carías, María Cristina, Vito Véliz y Ricardo Agurcia Fasquelle. Acerca de las observaciones del Sol realizadas en la Gran Plaza del Parque Arqueológico de Copán, Honduras. Yaxkin, XXI. Instituto Hondureño de Antropología e 
Historia. 2002.

Pineda de Carías, María Cristina, Vito Véliz y Ricardo Agurcia Fasquelle. El grande y complejo plan de 18 Conejo para la construcción de la Plaza del Sol del Parque Arqueológico de Copán, Honduras. Revista del Instituto Hondureño de Antropología e Historia. Edición Conmemorativa 50 Aniversario 1952-2002. Julio, 2002.

Silberschatz, Abraham, Henry F. Korth y S. Sudarshan. Fundamentos de Bases de Datos. 5 ed. McGraw Hill Interamericana de España, S.A. U. 2006.

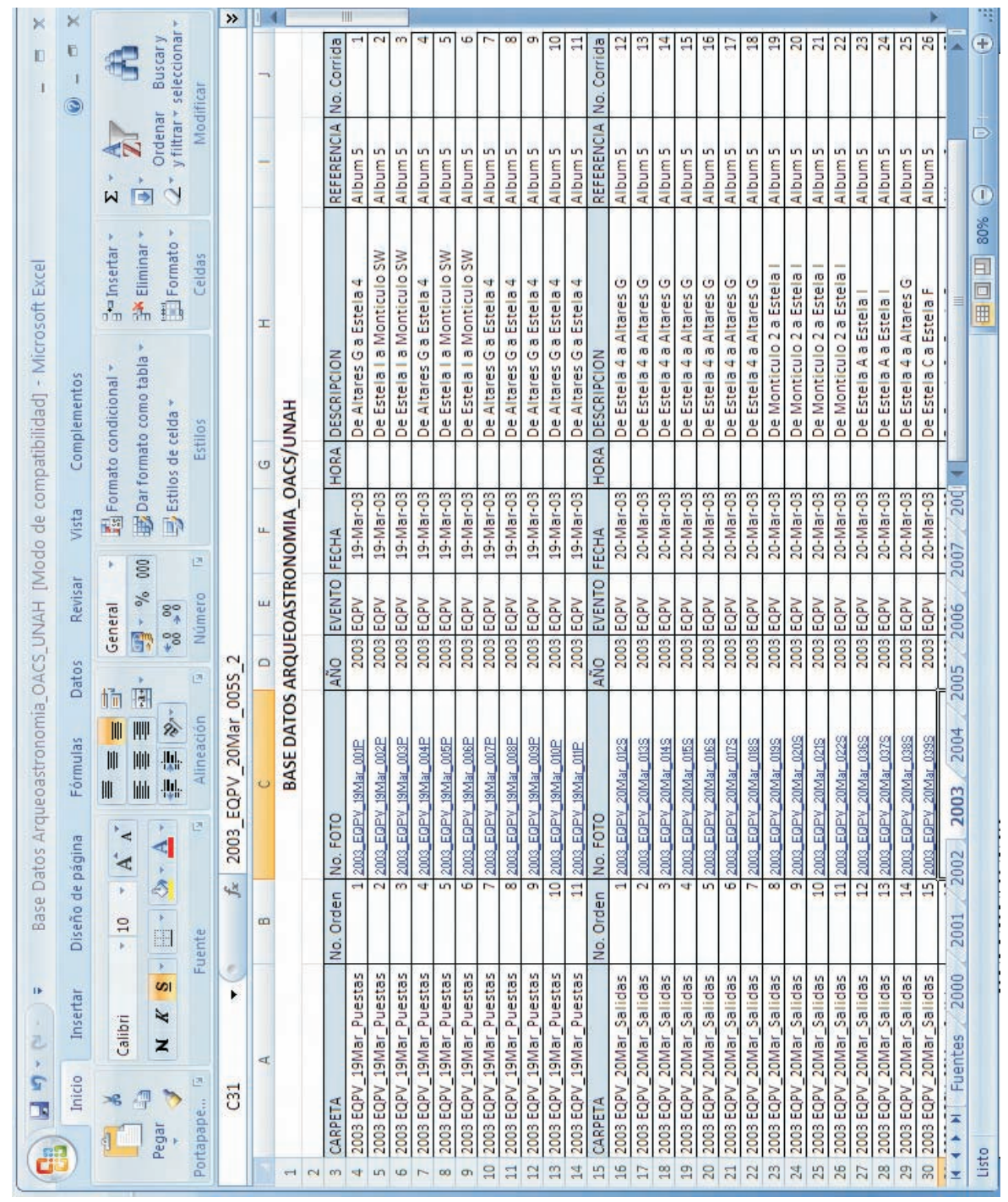

Figura 1. Tabla con atributos de fotografias tomadas en el año 2003 


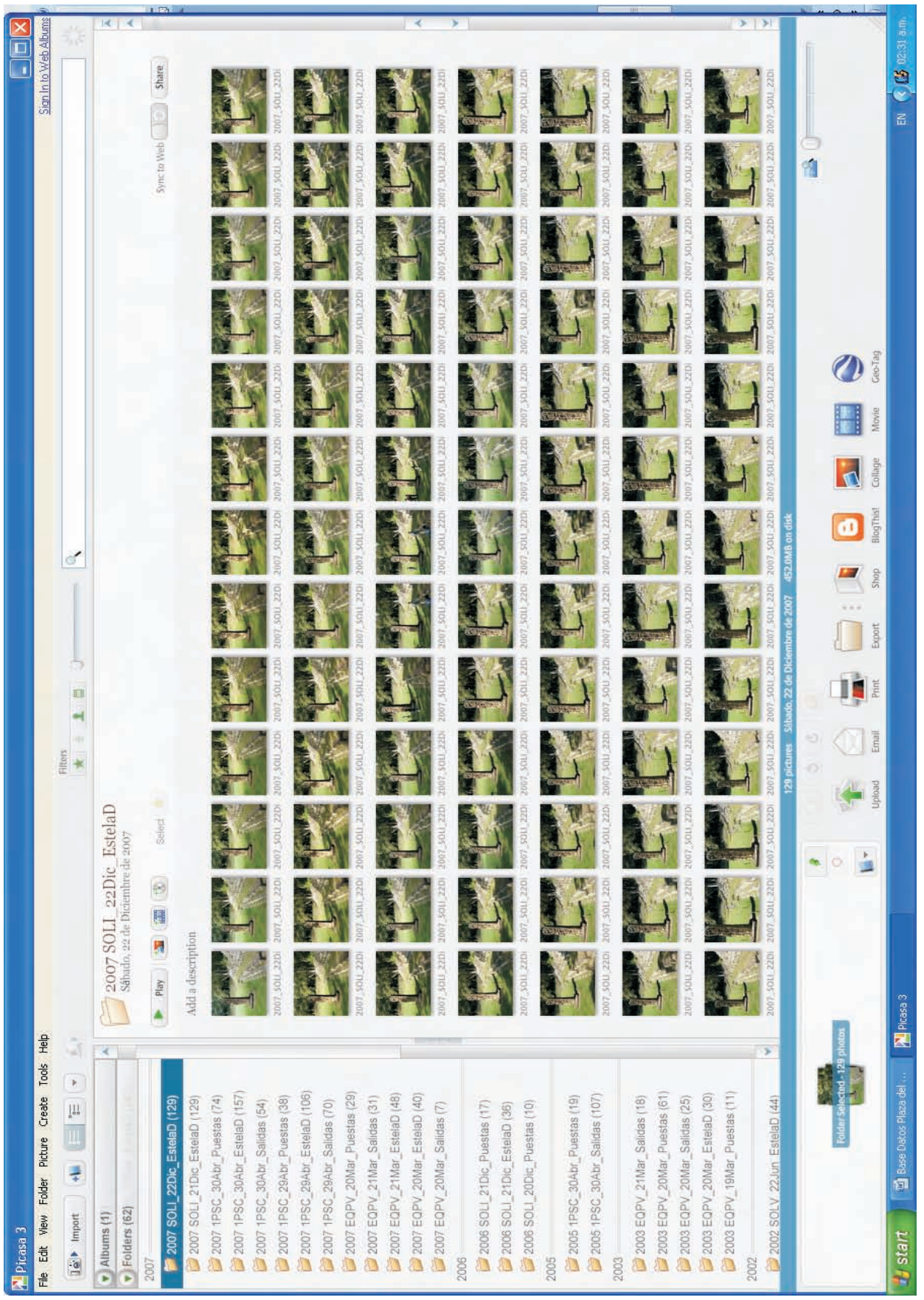

Figura 2. Pantalla de Picasa3 que muestra una vista del esquema de organización de la base de datos 


\section{ANEXO A.1}

\section{RESUMEN DE DATOS DE FOTOGRAFIAS SEGUN EVENTOS SOLARES Y FECHAS \\ Equinoccios de Primavera (EQPV)}

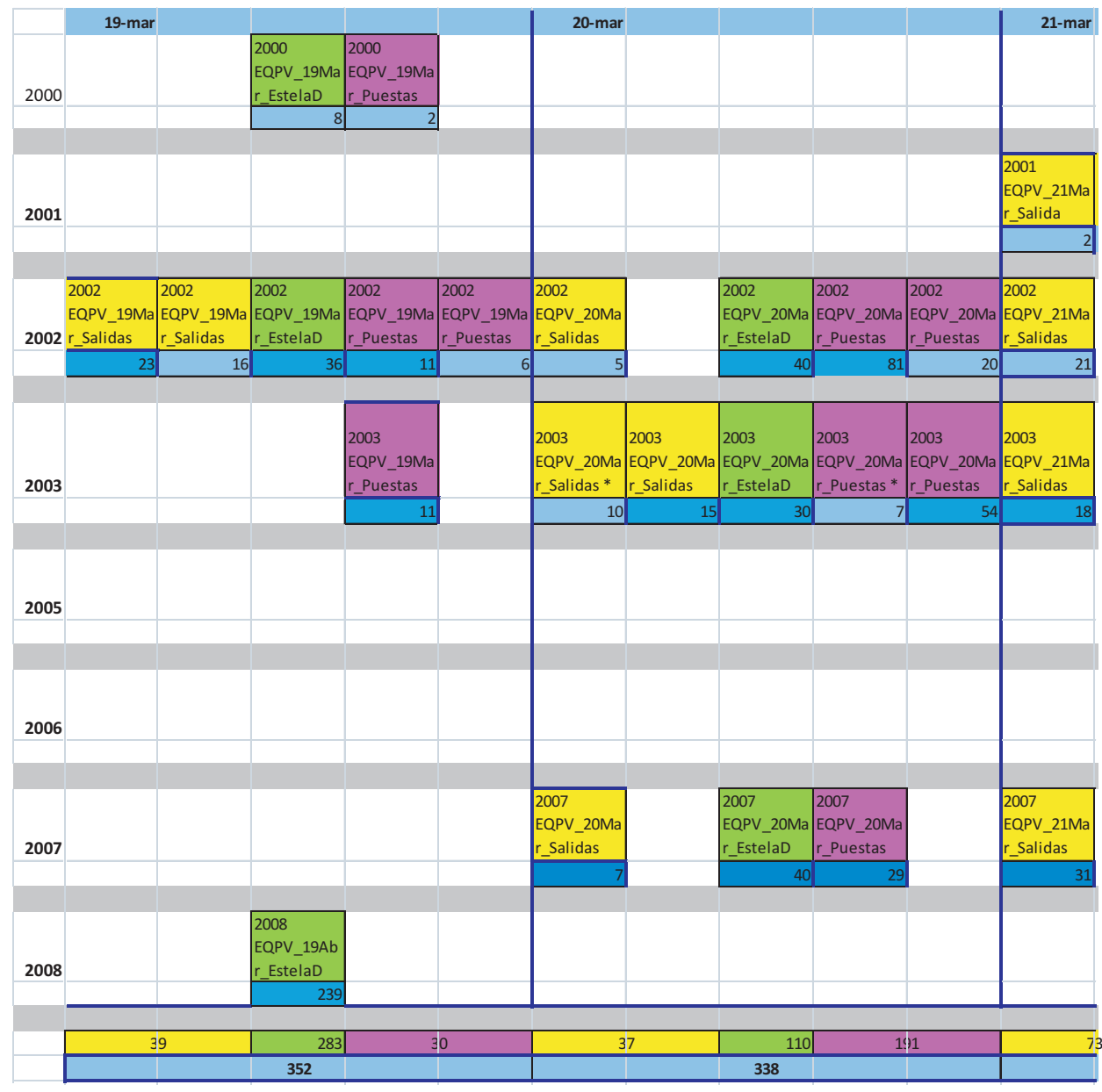

121 


\section{Primer Paso del Sol por el Cenit (1PSC)}

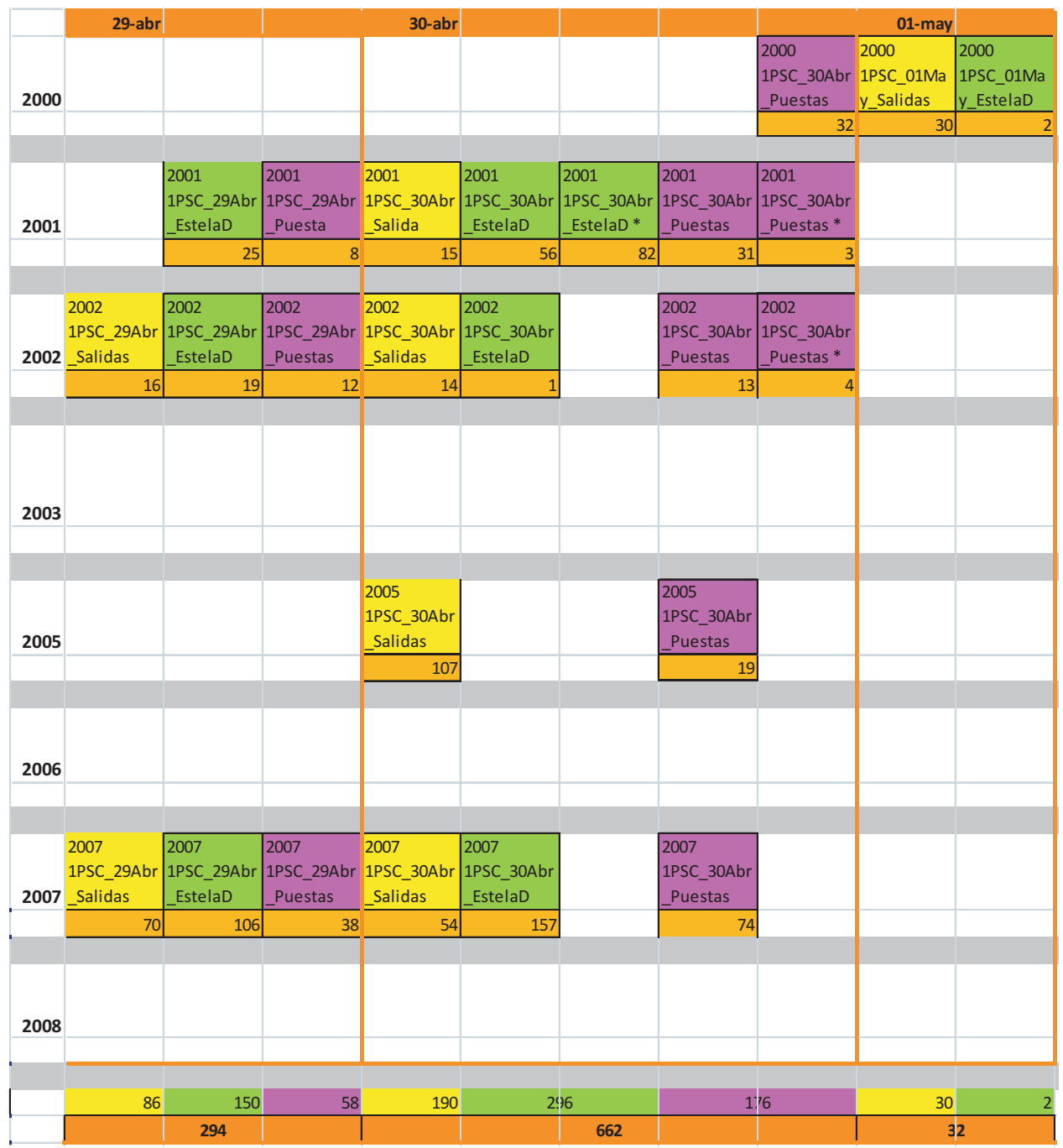




\section{SOLSTICIO DE VERANO (SOLV)}

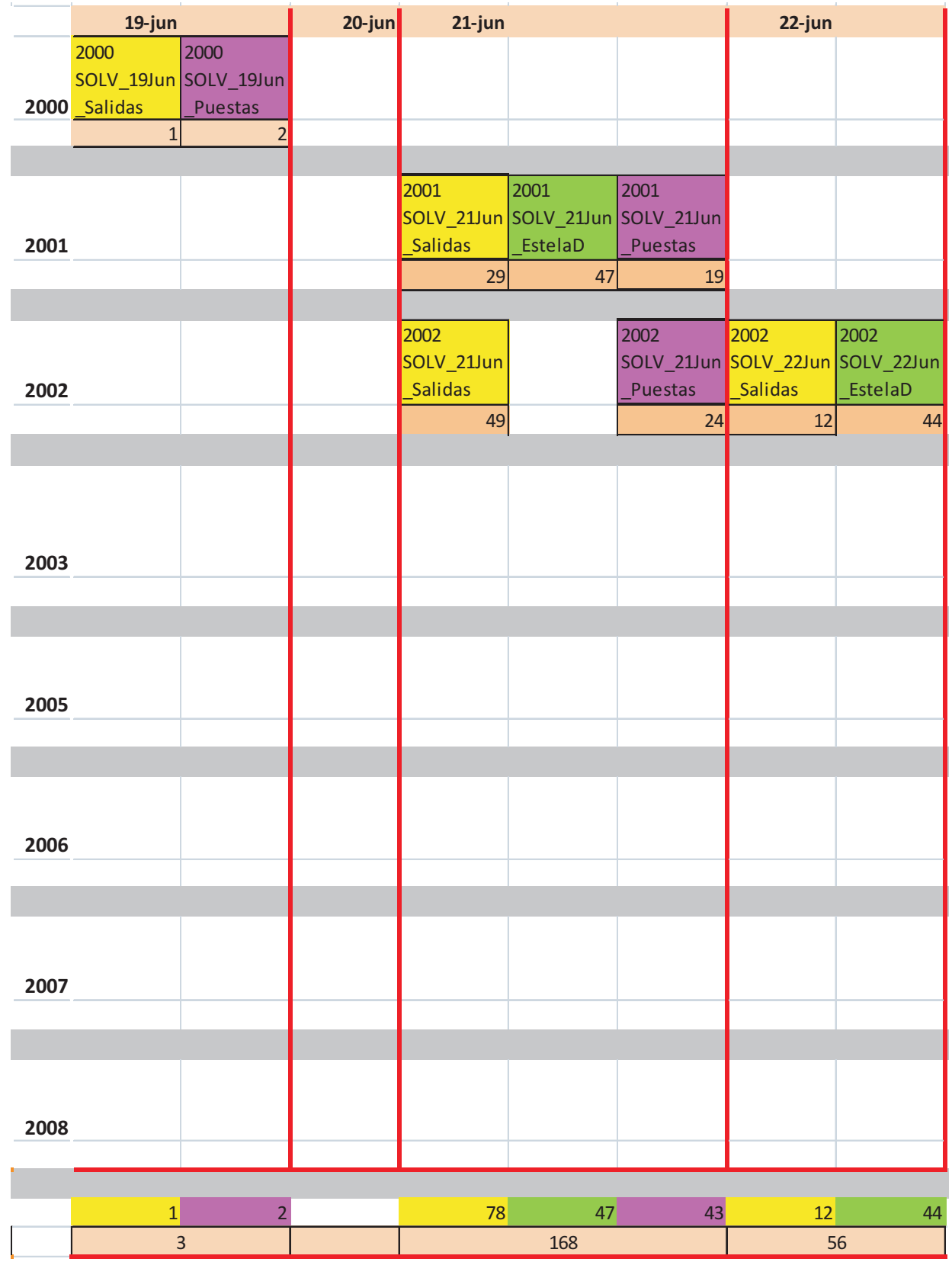

34 | Dirección de Investigación Científica 


\section{Segundo Paso del Sol por el Cenit (2PSC) y Solsticio de Invierno (SOLI)}

\begin{tabular}{|c|c|c|c|c|c|c|c|c|c|c|}
\hline 11-ago & 12 -ago & & & 20-dic & 21-dic & & & & & 22-dic \\
\hline & 2000 & 2000 & & & 2000 & 2000 & 2000 & 2000 & & \\
\hline & 2PSC_12Ago & 2PSC_12Ago & & & SOLI_21Dic_ & SOLI_21Dic_ & SOLI_21Dic_ & SOLI_21Dic_ & & \\
\hline & EstelaD & Puestas & 2000 & & Salidas2 & Salidas & EstelaD2 & EstelaD & & \\
\hline & 14 & 3 & & & 3 & 47 & 10 & 22 & & \\
\hline & & & & & & & & & & \\
\hline 2001 & & & & & & & & & & \\
\hline 2PSC_11Ago & & & & & & & & & & \\
\hline Puestas & & & 2001 & & & & & & & \\
\hline 21 & & & & & & & & & & \\
\hline & & & & & & & & & & \\
\hline & & & 2002 & & & & & & & \\
\hline & & & & & & & & & & \\
\hline & & & & & & & & & & \\
\hline & & & & & & & & & & \\
\hline & & & 2003 & & & & & & & \\
\hline & & & & & & & & & & \\
\hline & & & & & & & & & & \\
\hline & & & & & & & & & & \\
\hline & & & 2005 & & & & & & & \\
\hline & & & & & & & & & & \\
\hline & & & & & & & & & & \\
\hline & & & & 2006 & & & 2006 & & 2006 & \\
\hline & & & & SOLI_20Dic_ & & & SOLI 21Dic & & SOLI 21DIC & \\
\hline & & & 2006 & Puestas & & & EstelaD & & Puestas & \\
\hline & & & & 10 & & & 36 & & 17 & \\
\hline & & & & & & & & & & \\
\hline & & & & & & & 2007 & & & 2007_SOLI_ \\
\hline & & & & & & & SOLI_21Dic_ & & & 22Dic_Estel \\
\hline & & & 2007 & & & & EstelaD & & & \\
\hline & & & & & & & 129 & & & 129 \\
\hline & & & & & & & & & & \\
\hline & & & & & & & & & & \\
\hline & & & 2008 & & & & & & & \\
\hline & & & & & & & & & & \\
\hline & & & & & & & & & & \\
\hline 21 & 14 & 3 & & 10 & & 50 & & 97 & 17 & 129 \\
\hline 21 & 17 & 7 & & 10 & & & 264 & & & 129 \\
\hline
\end{tabular}

